\title{
MINIMAL BOUNDED INDEX SUBGROUP FOR DEPENDENT THEORIES
}

\author{
SAHARON SHELAH \\ (Communicated by Julia Knight)
}

\begin{abstract}
For a dependent theory $T$, in $\mathfrak{C}_{T}$ for every type definable group $G$, the intersection of type definable subgroups with bounded index is a type definable subgroup with bounded index.
\end{abstract}

\section{$\S 0$. INTRODUCTION}

Assume that $T$ is a dependent (complete first-order) theory, $\mathfrak{C}$ is a $\bar{\kappa}$-saturated model of $T$ (a monster), $G$ is a type definable (in $\mathfrak{C}$ ) group in $\mathfrak{C}$ (of course we consider only types of cardinality $<\bar{\kappa}$ ).

A type definable subgroup $H$ of $G$ is call bounded if the index $(G: H)$ is $<\bar{\kappa}$. We prove that there is a minimal bounded definable subgroup. The first theorem on this line for $T$ stable is due to Baldwin and Saxl [BaSx76].

Recently Hrushovski, Peterzil and Pillay [HPP0x] investigated definable groups, o-minimality and measure. In an earlier work on definable subgroups in o-minimal $T$ in Berarducci, Otero, Peterzil and Pillay [BOPP05] the minimal type-definable bounded index theorem and more results are proved for o-minimal theories.

Hrushovski, in a lecture at the Hebrew University, mentioned that he, Peterzil and Pillay had observed the main result of the current paper, but assuming in addition the existence of an invariant measure on the group in question, and Hrushovski asked if the measure assumption could be removed. So we answer it positively. The current version of their paper [HPP0x] includes an exposition of our proof.

Recent works of the author on dependent theories are [Sh:783] (see $\S 3, \S 4$ on groups) [Sh:863] (e.g., the first-order theory of the $p$-adics is strongly ${ }^{1}$ dependent but not strongly ${ }^{2}$ dependent, see end of $\S 1$; on strongly ${ }^{2}$ dependent fields, see $\S 5$ ) and [Sh:F705]. This work is continued in [Sh:F753] (getting mainly a parallel result for $G$ abelian and $\mathbb{L}_{\infty, \bar{\kappa}}$-definable subgroups).

I thank Aviv Tatarski and Ilay Kaplan for their very careful checking.

Received by the editors January 22, 2006 and, in revised form, July 19, 2006, July 30, 2006, and October 19, 2006.

2000 Mathematics Subject Classification. Primary 03C45; Secondary 03C60.

The author would like to thank the Israel Science Foundation for partial support of this research (Grant No. 242/03), Publication 876. I would like to thank Alice Leonhardt for the beautiful typing. 
1.1 Theorem. Assume $T$ is a dependent (complete first-order) theory, $\mathfrak{C}$ a $\bar{\kappa}$ saturated model for $T$. We consider types of cardinality $<\bar{\kappa}$.

1) If $\circledast$ below holds, then:

$(\alpha) q(\mathfrak{C})$ is a subgroup of $p(\mathfrak{C})$,

$(\beta) q(\mathfrak{C})$ is of index $<\bar{\kappa}$,

$(\gamma)$ essentially $q(x) \backslash p(x)$ is of cardinality $\leq \lambda:=|T|^{\aleph_{0}}$ (i.e., for some $q^{\prime}(x) \subseteq$ $q(x)$ of cardinality $\leq \lambda, q(x)$ is equivalent to $\left.p(x) \cup q^{\prime}(x)\right)$,

$(\delta)$ we can strengthen $(\alpha),(\beta)$ to $q(\mathfrak{C})$ is a subgroup of index $\leq 2^{\lambda}$, where

$\circledast(a) \quad p(x)$ is a type such that $p(\mathfrak{C})$ is a group which we call $G$ (with some definable operations $x y, x^{-1}$ and the identity $e_{G}$ which is constant here),

(b) $q(x)=p(x) \cup \bigcup\{r(x): r(x) \in \mathbf{R}\}$, where

(c) $\mathbf{R}=\{r(x): r(x)$ a type such that $(p \cup r)(\mathfrak{C})$ is a subgroup of $p(\mathfrak{C})$ of index $<\bar{\kappa}\}$.

2) There exists some $q^{\prime} \subseteq q$ over $\operatorname{Dom}(p)$, equivalent to $q$ and such that $\left|q^{\prime}\right| \leq$ $|T|+|\operatorname{Dom}(p)|$. So $(p(\mathfrak{C}): q(\mathfrak{C})) \leq 2^{|\operatorname{Dom}(p)|+|T|}$.

3) If $r_{i}(x) \in \mathbf{R}$ for $i<\lambda^{+}$, then for some $\alpha<\left(|T|^{\aleph_{0}}\right)^{+}$we have $\left(p(x) \cup \bigcup\left\{r_{i}(x)\right.\right.$ : $i<\alpha\})(\mathfrak{C})=\left(p(x) \cup \bigcup\left\{r_{i}(x): i<\lambda^{+}\right\}\right)(\mathfrak{C})$.

\section{Proof. 1) Note}

$\circledast_{1} \mathbf{R}$ is closed under unions of $<\bar{\kappa}$.

$\circledast_{2}(a) \quad$ If $r(x) \in \mathbf{R}, r^{\prime}(x) \subseteq r(x)$ is countable, then there is a countable $r^{\prime \prime}(x) \subseteq r(x)$ including $r^{\prime}(x)$ which belongs to $\mathbf{R}$.

(b) If $p(x) \subseteq r(x) \in \mathbf{R}$ and $r(x)$ is closed under conjunctions and $r^{\prime}(x) \subseteq$ $r(x)$ is countable, then we can find $\psi_{n}\left(x, \bar{b}_{n}\right)$ for $n<\omega$ such that

( $\alpha) \psi_{n}\left(x, \bar{b}_{n}\right) \in r(x)$,

( $\beta) \psi_{n+1}\left(x, \bar{b}_{n+1}\right) \vdash \psi_{n}\left(x, \bar{b}_{n}\right)$,

$(\gamma) \psi_{n+1}\left(x, \bar{b}_{n+1}\right), \psi_{n+1}\left(y, \bar{b}_{n+1}\right) \vdash \psi_{n}\left(x^{-1} y, \bar{b}_{n}\right) \wedge \psi_{n}\left(x^{-1}, \bar{b}_{n}\right) \wedge \psi_{n}\left(x y, \bar{b}_{n}\right)$,

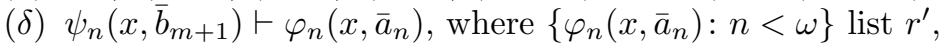

$(\varepsilon) \mathfrak{C} \models \psi_{n}\left(e_{G}, \bar{b}_{n}\right)$, actually follows from clause $(\alpha)$.

[Why? Let $r^{\prime}(x)=\left\{\varphi_{n}\left(x, \bar{a}_{n}\right): n<\omega\right\}$ (can use $\varphi_{n}=(x=x)$ ). Without loss of generality, $r(x)$ is closed under conjunctions and also $r^{\prime}(x)$ is. Now we choose $\psi_{n}\left(x, \bar{b}_{n}\right)$ by induction on $n<\omega$ such that $\psi_{n+1}\left(x, \bar{b}_{n+1}\right) \wedge \psi_{n+1}\left(y, \bar{b}_{n+1}\right) \vdash \psi_{n}\left(x y^{-1}, \bar{b}_{n}\right) \wedge \varphi_{n}\left(x, \bar{a}_{n}\right) \wedge \psi_{n}\left(x y, \bar{b}_{n}\right) ;$ notice that trivially $e_{G} \in \varphi_{n}\left(\mathfrak{C}, \bar{a}_{n}\right) \cap \psi_{n}\left(\mathfrak{C}, \bar{b}_{n}\right)$. Such a formula exists as $(p(x) \cup r(x)) \cup(p(y) \cup r(y)) \vdash \psi_{n}\left(x y^{-1}, \bar{b}_{n}\right) \wedge \varphi_{n}\left(x, \bar{a}_{n}\right) \wedge \psi_{n}\left(x y, \bar{b}_{n}\right)$.

Now $r^{\prime \prime}(x)=\left\{\varphi_{n}\left(x, \bar{a}_{n}\right), \psi_{n}\left(x, \bar{b}_{n}\right): n<\omega\right\}$ is as required in clause (a), $\left\langle\psi_{n}\left(x, \bar{b}_{n}\right): n<\omega\right\rangle$ in $(b)$.]

In the conclusion of Theorem 1.1, Clause $(\alpha)$ is obvious.

Assume toward a contradiction that the conclusion $(\beta)+(\gamma)$ fails. So we can choose $\left(c_{\alpha}, r_{\alpha}\right)$ by induction on $\alpha<\lambda^{+}$such that

$$
\begin{aligned}
& \circledast_{3} \quad \text { (a) } c_{\alpha} \in\left(p(x) \cup \bigcup\left\{r_{\beta}: \beta<\alpha\right\}\right)(\mathfrak{C}), \\
& \text { (b) } r_{\alpha}=\left\{\psi_{n}^{\alpha}\left(x, \bar{b}_{n}^{\alpha}\right): n<\omega\right\} \subseteq q \text { and } \bar{b}_{n}^{\alpha} \triangleleft \bar{b}_{n+1}^{\alpha}, \\
& \text { (c) } r_{\alpha} \in \mathbf{R} \text {, and } \psi_{n+1}\left(x, \bar{b}_{n+1}^{\alpha}\right) \vdash \psi_{n}\left(x, \bar{b}_{n}^{\alpha}\right), \\
& \text { (d) } c_{\alpha} \text { does not realize } r_{\alpha} \text {, in fact } \mathfrak{C} \models \neg \psi_{0}^{\alpha}\left(c_{\alpha}, \bar{b}_{0}^{\alpha}\right) \text {, hence } c_{\alpha} \notin q(\mathfrak{C}) .
\end{aligned}
$$


[Why? Arriving to $\alpha$, we try to let $q_{\alpha}=p(x) \cup \bigcup\left\{r_{\beta}: \beta<\alpha\right\}$, so $q_{\alpha} \subseteq q$ and by cardinality considerations, $q_{\alpha}(\mathfrak{C})$ is a subgroup of $G$ of index $<\bar{\kappa}$. So by our assumption toward a contradiction, $q_{\alpha}(x) \nVdash q(x)$; hence there is $r_{\alpha}^{*}(x) \in \mathbf{R}$ such that $q_{\alpha}(x) \nVdash r_{\alpha}^{*}$, so $q_{\alpha}(x) \nVdash \vartheta_{\alpha}\left(x, \bar{d}_{\alpha}\right)$ for some $\vartheta_{\alpha}\left(x, \bar{d}_{\alpha}\right) \in$ $r_{\alpha}^{*}$. Let $r_{\alpha}(x)=\left\{\psi_{n}^{\alpha}\left(x, \bar{b}_{n}^{\alpha}\right): n<\omega\right\} \subseteq r_{\alpha}^{*}(x)$ belong to $\mathbf{R}$ and be such that $\vartheta_{\alpha}\left(x, \bar{d}_{\alpha}\right)=\psi_{0}^{\alpha}\left(x, \bar{b}_{0}^{\alpha}\right)$; it exists by $\circledast_{2}(\alpha)$ above.

Without loss of generality, we can assume $\circledast_{4}-\circledast_{8}$ :

$\circledast_{4} e_{G}$ is an individual constant, $\bar{y}_{n}^{\alpha} \unlhd \bar{y}_{n+1}^{\alpha}$ and $\psi_{n+1}^{\alpha}\left(x, \bar{y}_{n+1}^{\alpha}\right) \vdash \psi_{n}^{\alpha}\left(x, \bar{y}_{n}^{\alpha}\right)$ and $\left(\psi_{n+1}^{\alpha}\left(x_{1}, \bar{y}_{n+1}^{\alpha}\right) \wedge \psi_{n+1}^{\alpha}\left(x_{2}, \bar{y}_{n+1}^{\alpha}\right)\right) \vdash\left(\psi_{n}^{\alpha}\left(e_{G}, \bar{y}_{n}^{\alpha}\right) \wedge \psi_{n}^{\alpha}\left(x_{1} x_{2}^{-1}, \bar{y}_{n}^{\alpha}\right)\right)$.

[Why? As in the proof of $\circledast_{2}$ above, i.e., during the induction in the proof of $\circledast_{3}$, we use $\circledast_{2}(\beta)$ and get $\psi_{n}^{\alpha}\left(x, \bar{y}_{n}^{\alpha}\right), \bar{b}_{n}^{\alpha}$ and without loss of generality $\bar{b}_{n}^{\alpha} \unlhd \bar{b}_{n+1}^{\alpha}, \bar{y}_{n}^{\alpha} \triangleleft \bar{y}_{n+1}^{\alpha}$ (as we can change the order and name the free variable and add dummy variables). Now we define $\psi_{n}^{\alpha, *}\left(x, \bar{y}_{n}^{\alpha}\right)$ by induction on $n$ by

$$
\begin{aligned}
& \psi_{0}^{\alpha, *}\left(x, \bar{y}_{0}^{\alpha}\right)=\psi_{0}^{\alpha}\left(x, \bar{y}_{0}^{\alpha}\right), \psi_{n+1}^{\alpha, *}\left(x, \bar{y}_{n+1}^{\alpha}\right)=\psi_{n+1}^{\alpha}\left(x, \bar{y}_{n+1}^{\alpha}\right) \\
& \wedge \bigwedge_{m \leq n}(\forall z)\left[\psi_{m+1}^{\alpha}\left(z, \bar{y}_{m+1}^{\alpha}\right) \rightarrow \psi_{m}^{\alpha}\left(z, \bar{y}_{m}^{\alpha}\right)\right] \\
& \wedge \bigwedge_{m \leq n}\left(\forall z_{1}, z_{2}\right)\left[\psi_{m+1}^{\alpha}\left(z_{1}, \bar{y}_{m+1}^{\alpha}\right) \wedge \psi_{m+1}^{\alpha}\left(z_{2}, \bar{y}_{m+1}^{\alpha}\right)\right. \\
&\left.\rightarrow \psi_{m}^{\alpha}\left(e_{G}, \bar{y}_{m}^{\alpha}\right) \wedge \psi_{m}^{\alpha}\left(z_{1} z_{2}^{-1}, \bar{y}_{m}^{\alpha}\right)\right] .
\end{aligned}
$$

So clearly $\left\langle\psi_{n}^{\alpha, *}\left(x, \bar{y}_{n}^{\alpha}\right): n<\omega\right\rangle$ satisfies $\circledast_{4}$ and $\mathfrak{C} \models(\forall x)\left[\psi_{n}^{\alpha, *}\left(x, \bar{b}_{n}^{\alpha}\right) \equiv\right.$ $\left.\psi_{n}^{\alpha}\left[x, \bar{b}_{n}^{\alpha}\right)\right]$. So renaming we are done. $]$

$\circledast_{5} \quad \psi_{n}^{\alpha}\left(x, \bar{y}_{n}^{\alpha}\right)=\psi_{n}\left(x, \bar{y}_{n}\right)$ and $\left.\psi_{n+1}\left(x, \bar{y}_{n+1}\right)\right) \vdash \psi_{n}\left(x, \bar{y}_{n}\right)$.

[Why? By the pigeon hole principle.]

$\circledast_{6}\left\langle c_{\alpha} \overline{\mathbf{a}}_{\alpha}: \alpha<\lambda^{+}\right\rangle$is an indiscernible sequence over $\operatorname{Dom}(p)$ where $\overline{\mathbf{a}}_{\alpha}=$ $\cup\left\{b_{n}^{-\alpha}: n<\omega\right\} \bar{b}_{0}^{\alpha \wedge} \bar{b}_{1}^{\alpha \wedge} \bar{b}_{2}^{\alpha \wedge} \ldots ;$ note that by clause (b) of $\circledast_{3}$ we have $\bar{b}_{n}^{\alpha}=\overline{\mathbf{a}}_{\alpha}\left\lceil k_{n}\right.$.

[Why? By the Ramsey theorem and compactness.]

$\circledast_{7}$ If $\alpha<\beta<\gamma$, then $c_{\alpha} c_{\beta}^{-1} \in r_{\gamma}(\mathfrak{C})$.

[Why? By the indiscernibility, without loss of generality, $\gamma$ is infinite, so $\gamma \geq$ $\omega$ and $\left\langle c_{i}: i<\gamma\right\rangle$ is an indiscernible sequence over $\operatorname{Dom}(p) \cup \bar{b}_{\gamma}$ of elements of $p(\mathfrak{C})$ pairwise nonequivalent modulo the subgroup $G_{\gamma}=\left(p \cup r_{\gamma}\right)(\mathfrak{C})$. Then we can extend it to $\left\langle\bar{c}_{i}: i<\bar{\kappa}\right\rangle$, an indiscernible sequence over $\operatorname{Dom}(p) \cup \bar{b}_{\gamma}$ and arrive at $\alpha<\beta \Rightarrow c_{\alpha} c_{\beta}^{-1} \notin G_{\gamma} \Rightarrow c_{\beta} c_{\alpha}^{-1} \notin G_{\gamma}$ so $\left\langle c_{\alpha} G_{\gamma}: \alpha<\bar{\kappa}\right\rangle$ are pairwise distinct (equivalently $\left\langle G_{\gamma} c_{\alpha}: \alpha<\bar{\kappa}\right\rangle$ are pairwise distinct), a contradiction.]

$\circledast_{8} \quad c_{\alpha} \in r_{\beta}(\mathfrak{C})$ iff $\alpha \neq \beta$.

[Why? Let

$$
\begin{gathered}
c_{\alpha}^{*}=c_{2 \alpha+1} \cdot\left(c_{2 \alpha}\right)^{-1}, \\
r_{\alpha}^{*}=r_{2 \alpha} .
\end{gathered}
$$

So:

(i) If $\beta<\alpha$, then $c_{\alpha}^{*} \in\left(p \cup r_{\beta}^{*}\right)(\mathfrak{C})$, because $c_{2 \alpha+1}, c_{2 \alpha}$ belong to the subgroup $\left(p \cup r_{2 \beta}\right)(\mathfrak{C})$ by clause $(\mathrm{a})$ of $\circledast_{3}$.

(ii) If $\beta>\alpha$, then $c_{\alpha}^{*}$ belongs to $\left(p \cup r_{\beta}^{*}\right)(\mathfrak{C})$ by $\circledast_{7}$.

(iii) If $\beta=\alpha$, then $c_{\alpha}^{*}$ does not belong to $\left(p \cup r_{\beta}^{*}\right)(\mathfrak{C})$ because: 
$(\alpha)$ it is a subgroup,

( $\beta) \quad c_{2 \alpha+1}$ belongs to it by clause (a) of $\circledast_{3}$ and

$(\gamma) \quad c_{2 \alpha}$ does not belong to it by clause $(\mathrm{e})$ of $\circledast_{3}$.

Let $\overline{\mathbf{a}}_{\alpha}^{*}=\overline{\mathbf{a}}_{2 \alpha+1}, \bar{b}_{n}^{\alpha, *}=\bar{b}_{n}^{2 \alpha}$ retaining the same $\psi$ 's. So we have obtained an example as required in $\circledast_{8}$ (not losing the other demands).]

$\circledast_{9}$ For some $n<\omega$ for every $\alpha$, we have if $d_{1}, d_{2} \in\left(p \cup r_{\alpha}\right)(\mathfrak{C})$, then $d_{1} c_{\alpha} d_{2} \notin \psi_{n}\left(\mathfrak{C}, \bar{b}_{n}^{\alpha}\right)$; without loss of generality, $n=1$.

[Why? Fix $\alpha$. If this holds for some $\psi_{n}\left(-, \bar{b}_{n}^{\alpha}\right)$, by indiscernibility, renaming the $\varphi_{i}$ 's, this is O.K. Otherwise for each $n<\omega$ there are $d_{1}^{n}, d_{2}^{n} \in\left(p \cup r_{\alpha}\right)(\mathfrak{C})$ such that $\mathfrak{C} \models \psi_{n}\left(d_{1}^{n} c_{\alpha} d_{2}^{n}, \bar{b}_{n}^{\alpha}\right)$. By compactness for some $d_{1}^{*}, d_{2}^{*} \in\left(p \cup r_{\alpha}\right)(\mathfrak{C})$ we have $\models \psi_{n}\left[d_{1}^{*} c_{\alpha} d_{2}^{*}, \bar{b}_{n}^{\alpha}\right]$ for every $n<\omega$. So $d_{1}^{*} c_{\alpha} d_{2}^{*}$ belongs to the subgroup $\left(p \cup r_{\alpha}\right)(\mathfrak{C})$, but also $d_{1}^{*}, d_{2}^{*}$ belongs to it; hence $c_{\alpha}$ belongs, a contradiction. Alternatively, note that $n=2$ is O.K.: let $c^{\prime}=d_{1} c d_{2}$ and assume toward a contradiction that $\left.c^{\prime} \in \psi_{2}\left(\mathfrak{C}, \bar{b}_{\alpha}\right)\right)$ and let $d_{1}^{\prime}=\left(d_{1}\right)^{-1}, d_{2}^{\prime}=\left(d_{2}\right)^{-1}$, so clearly $d_{1}, d_{2}^{\prime} \in\left(p \cup r_{\alpha}\right)(\mathfrak{C}) \subseteq \psi_{2}\left(\mathfrak{C}, \bar{b}_{2}^{\alpha}\right)$. Now by $\circledast_{4}$ as $d_{1}^{\prime}, c^{\prime} \in \psi_{2}\left(\mathfrak{C}, \bar{b}_{\alpha_{1}}^{\alpha}\right)$ it follows that $d_{2}\left(c^{\prime}\right)^{-1} \in \psi_{1}\left(\mathfrak{C}, \bar{b}_{1}^{\alpha}\right)$. As $d_{1}, d_{2}^{\prime}\left(c^{\prime}\right)^{-1} \in \psi_{1}\left(\mathfrak{C}, \bar{b}_{1}^{\alpha}\right)$ by $\circledast_{4}$ we have $d_{1}^{\prime}\left(d_{2}\left(c^{\prime}\right)^{-1}\right)^{-1} \in \psi_{0}\left(\mathfrak{C}^{\mathfrak{c}}, \bar{b}_{0}^{\alpha}\right)$. But $c^{\prime}=d_{1} c_{\alpha} d_{2}$; hence $c_{\alpha}=$ $d_{1}^{\prime}\left(\left(d_{2}^{\prime}\right)^{-1}\left(c^{1}\right)^{-1}\right)^{-1}=d_{1}^{\prime}\left(d_{2}\left(c^{\prime}\right)^{-1}\right)^{-1}$, but $d_{1}^{\prime}\left(d_{2}\left(c^{\prime}\right)^{-1}\right)^{-1} \in \psi_{0}\left(\mathfrak{C}, \bar{b}_{0}^{\alpha}\right)$ by the previous sentence, whereas $c_{\alpha} \notin \psi_{0}\left(\mathfrak{C}, \bar{b}_{1}^{\alpha}\right)$ by $\circledast_{3}(e)$, a contradiction.]

$\circledast 10$ If $w=\left\{i_{1}, \ldots, i_{n}\right\}, i_{1}<\ldots<i_{n}<\lambda^{+}$, and $d_{w}:=c_{i_{1}} c_{i_{2}} \ldots c_{i_{n}} \in G$ and $\alpha<\lambda^{+}$, then $\models \varphi_{1}\left[d_{w}, \bar{b}_{\alpha}^{1}\right] \Leftrightarrow \alpha \notin w$.

[Why? If $\alpha \in w$, let $k$ be such that $\alpha=i_{k}$, so $c_{i_{1}}, \ldots, c_{i_{k-1}} \in\left(p \cup r_{\alpha}\right)(\mathfrak{C})$ by $\circledast_{8}$ and similarly $c_{i_{k+1}} \ldots c_{i_{n}} \in\left(p \cup r_{\alpha}\right)(\mathfrak{C})$; hence

$$
d_{w}=\left(c_{i_{1}} \ldots c_{i_{k}}\right) c_{i_{k}}\left(c_{i_{k+1}} \ldots c_{i_{n}}\right) \notin\left(p \cup r_{\alpha}\right)(\mathfrak{C})
$$

by $\circledast 9$.

Second, if $\alpha \notin w$, this holds by $\circledast_{8}$ as $\left\{c_{i_{\ell}}: \ell<n\right\}$ is included in the subgroup $\left(p \cup r_{\alpha}\right)(\mathfrak{C})$.]

So we get a contradiction to " $T$ is dependent"; hence clauses $(\beta),(\gamma)$ hold. Also clause $(\delta)$ follows by the following observation:

Observation: If $r(x) \in \mathbf{R}$ and $|r(x)| \leq \theta$, then $(p(\mathfrak{C}):(p \cup r)(\mathfrak{C})) \leq 2^{\theta}$ (except for being just finite when $\theta$ is finite).

Proof. If $\theta$ is finite, then the proof follows by compactness. If $\theta$ is infinite, then without loss of generality, $r$ is closed under conjunctions. Let $r=\left\{\varphi_{i}(x, \overline{\mathbf{b}}): i<\theta\right\}$, where $\overline{\mathbf{b}}$ is possibly infinite.

Let $u$ be a set of ordinals $(<\bar{\kappa})$ such that $\bar{\kappa}>|u|>(p(\mathfrak{C}):(p \cup r)(\mathfrak{C}))$. Now for each $i<\theta$, let $\Gamma_{i, u}=\bigcup\left\{p\left(x_{\alpha}\right): \alpha \in u\right\} \cup\left\{\neg \varphi_{i}\left(x_{\alpha} x_{\beta}^{-1}, \overline{\mathbf{b}}\right): \alpha<\beta\right.$ from $\left.u\right\}$. So for some finite $u_{i}^{*} \subseteq u, \Gamma_{i, u_{i}^{*}}$ is contradictory, so $\Gamma_{i, n_{i}}$ is contradictory, when $n_{i}=\left|u_{i}\right|$. It suffices to use $\left(2^{\theta}\right)^{+} \rightarrow\left(\ldots n_{i} \ldots\right)_{i<\theta}$ (why? let $\left\langle c_{\alpha}: \alpha<\left(2^{\theta}\right)^{+}\right\rangle$exemplify the failure and let $\left.\zeta_{\alpha, \beta}=\operatorname{Min}\left\{i: \mid=\neg \varphi_{i}\left(c_{\alpha} c_{\beta}^{-1}, \overline{\mathbf{b}}\right)\right\}\right)$.

This finishes the proof of part (1). We still need to prove 2), 3).

2) Let $q^{\prime}(x) \subseteq q(x)$ have cardinality $\leq|T|^{\aleph_{0}}$ and be such that $q(\mathfrak{C})=\left(p \cup q^{\prime}\right)(\mathfrak{C})$; $q^{\prime}(x)$ exists by part (1). Observe that every automorphism of $\mathfrak{C}$ fixing $\operatorname{Dom}(p)$ maps $p(\mathfrak{C})$ onto itself and therefore maps $q(\mathfrak{C})$ onto itself. 
It follows that if $c_{1}, c_{2} \in p(\mathfrak{C})$ are such that $\operatorname{tp}\left(c_{1}, \operatorname{Dom}(p)\right)=\operatorname{tp}\left(c_{2}, \operatorname{Dom}(p)\right)$, then $c_{1} \in q(\mathfrak{C})$ if and only if $c_{2} \in q(\mathfrak{C})$. Let $\mathbf{P}:=\{\operatorname{tp}(b, \operatorname{Dom}(p)): b \in q(\mathfrak{C})\}, \mathbf{P}(\mathfrak{C}):=$ $\bigcup\{r(\mathfrak{C}): r \in \mathbf{P}\}$. Then by the above explanation, $\mathbf{P}(\mathfrak{C}) \subseteq q(\mathfrak{C})$. By definition, $q(\mathfrak{C}) \subseteq p(\mathfrak{C})$, so they are equal. Let $q_{* *}=\bigcap\{r: r \in \mathbf{P}\}$, so we have $q(\mathfrak{C}) \subseteq q_{* *}(\mathfrak{C})$.

If they are equal, then we are done. Otherwise take $c_{1} \in q_{* *}(\mathfrak{C}) \backslash q(\mathfrak{C})$. Without loss of generality, let $\psi(x, \bar{d}) \in q$ be such that $\models \neg \psi\left(c_{1}, \bar{d}\right)$.

By definition of $\mathbf{P}$ and $c_{1}$, for each $\theta(x, \bar{e}) \in \operatorname{tp}\left(c_{1}, \operatorname{Dom}(p)\right)$ there exists some $p_{\theta(x, \bar{e})} \in \mathbf{P}$ such that $\theta(x, \bar{e}) \in p_{(x, \bar{e})}$ and therefore some $c_{\theta(x, \bar{e})} \in q(\mathfrak{C})$ satisfies $\theta(x, \bar{e})$. So $\operatorname{tp}\left(c_{1}, \operatorname{dom}(p)\right) \cup q^{\prime}(x)$ is finitely satisfiable and is therefore realized by some $c_{2}$. Thus $\operatorname{tp}\left(c_{1}, \operatorname{Dom}(p)\right)=\operatorname{tp}\left(c_{2}, \operatorname{Dom}(p)\right)$, but $c_{1} \notin q^{\prime}(\mathfrak{C})=q(\mathfrak{C})$ and $c_{2} \in\left(p \cup q^{\prime}\right)(\mathfrak{C})$, a contradiction.

3) By the proof of part (1).

\section{REFERENCES}

[BaSx76] John T. Baldwin and Jan Saxl. Logical stability in group theory. J. Austral. Math. Soc. Ser. A, 21:267-276, 1976. MR0407151 (53:10934)

[BOPP05] Alessandro Berarducci, Margarita Otero, Yaa'cov Peterzil, and Anand Pillay. A descending chain condition for groups definable in o-minimal structures. Annals of Pure and Applied Logic, 134:303-313, 2005. MR2139910 (2006a:03052)

[HPP0x] Ehud Hrushovski, Ya'acov Peterzil, and Anand Pillay. Groups, measures, and the NIP. preprint, 2005.

[Sh:F753] Saharon Shelah. Definable Groups for Dependent Theories.

[Sh:783] Saharon Shelah. Dependent first order theories, continued. Israel Journal of Mathematic, accepted. math.LO/0406440.

[Sh:863] Saharon Shelah. Strongly dependent theories. Israel Journal of Mathematics, submitted. math.LO/0504197.

[Sh:F705] Shelah, Saharon. Representation over orders of elementary classes.

The Hebrew University of Jerusalem, Einstein Institute of Mathematics, Edmond J. Safra Campus, Givat Ram, Jerusalem 91904, Israel

Current address: Department of Mathematics, Hill Center-Busch Campus, Rutgers, The State University of New Jersey, 110 Frelinghuysen Road, Piscataway, New Jersey 08854-8019 